\title{
BLACK HOLES IN THE UNIVERSE
}

\author{
I. D. NOVIKOV \\ Theoretical Astrophysics Center, Juliane Maries Vej 30, DK-2100 Copenhagen $\emptyset$, \\ Denmark; \\ University Observatory, Juliane Maries Vej 30, DK-2100 Copenhagen Ø, Denmark; \\ Astro Space Centre of P.N. Lebedev Physical Institute, \\ Profsoyuznaya 84/32, Moscow, 117810, Russia; \\ Nordita, Blegdamsvej 17, DK-2100 Copenhagen $\emptyset$, Denmark.
}

\section{Introduction}

Some 30 years ago very few scientists thought that black holes may really exist. Attention focussed on the black hole hypothesis after neutron stars had been discovered. It was rather surprising that astrophysicists immediately 'welcomed' black holes. They found their place not only in the remnants of supernova explosions but also in the nuclei of galaxies and quasars.

Of all conceptions of the human mind perhaps the most fantastic is the black hole. Black holes are neither bodies nor radiation. They are clots of gravity. The study of black hole physics extends our knowledge of the fundamental properties of space and time. Quantum processes occur in the neighborhood of black holes, so that the most intricate structure of the physical vacuum is revealed. Even more powerful (catastrophically powerful) quantum processes take place inside black holes (in the vicinity of the singularity).

It may be said that black holes are a door to a new, very wide field of study of the physical world.

In this paper I will give a brief review of some problems of physics and astrophysics of black holes. For systematic discussion of the problems see the books: Thorne et al. (1986), Novikov and Frolov (1989), section "Black Holes" in the book Kawaler et al. (1997), and Frolov and Novikov (1998).

Let us start from physics.

A black hole is region in space-time from which no signal can escape to an external observer. A black hole's boundary is a so called event horizon.

After the gravitational collapse of a celestial body and formation of a black hole the external gravitational field of it asymptotically approaches a standard equilibrium configuration known as the Kerr-Newman field and characterized by just three numbers: mass, angular momentum and charge.

\section{Physics outside a black hole}

Black holes reside in curved space. If a black hole has nonzero angular momentum then anything near a black hole will be dragged along by the vortex gravitational field. In this section I will consider a black hole without electric charge (Kerr black hole). The horizon's surface area can be written in terms of its mass $M$ and angular momentum $J=a M$, where $a$ is an angular momentum per unit mass $(c=1, G=1)$ :

$$
\begin{gathered}
A=4 \pi\left(r_{H}^{2}+a^{2}\right), \\
r_{H}=M+\sqrt{M^{2}-a^{2}} .
\end{gathered}
$$

The rotational energy, or corresponding mass $M_{\text {rot }}$, of a Kerr black hole is the following

$$
M_{\text {rot }}=M-\left[\frac{1}{2} M\left(M+\sqrt{M^{2}-a^{2}}\right)\right]^{1 / 2} \text {. }
$$


This rotational energy (energy of the vortex gravitational field) can be extracted (in principle) from a black hole.

The black hole is a clot of gravity, there is not any real matter on the horizon. In spite of this fact the horizon looks for an external observer (outside the black hole) and behaves as a physical membrane which is made from a two-dimensional viscous fuid with definite mechanical, electrical and thermodynamic properties.

This remarkable viewpoint is known as the membrane paradigm (see Thorne et al. (1986) for a review). According to this paradigm the interaction of the horizon with the external universe is described in terms of familiar laws for the horizon fluid, e.g. the Navier-Stokes equation, Maxwell's equations, a tidal force equation, and the equations of thermodynamics. It is very important to emphasize that the membrane paradigm is not an approximation method or some analogy. It is an exact formalism which gives exactly the same results as the standard formalism of the General Relativity. Because the laws governing the horizon's behavior have familiar forms, they are powerful for understanding intuitively and computing quantitatively the interaction of black holes with complex environments.

In subsequent parts of this section we will consider some manifestations of the physical properties of the black hole's membrane, that resided in the three dimensional space.

\subsection{MECHANICAL PROPERTIES OF THE HORIZON'S MEMBRANE}

According to the membrane formalism, from the point of view of an external observer the hole's membrane has definite surface mass density and the surface pressure and viscosity.

The formula for the mass density is

$$
\sum=-\frac{1}{8 \pi} \theta^{H}, \quad \theta^{H} \equiv \frac{d(\Delta A)}{\Delta A d t},
$$

where $\theta^{H}$ is a fractional change of area of a surface element per unit time of an observer at infinity. One can see that for the case of a black hole in equilibrium (for example, a nonrotating (Schwarzschild) or a Kerr hole in the empty space) $\sum=0$. The value of $\theta^{H}$ is always non-negative, consequently $\sum$ is always non-positive.

There is surface pressure $p^{H}$ in the membrane. For a Schwarzschild hole it is:

$$
p^{H}=\frac{1}{32 \pi M} \approx\left(10^{42} \frac{\text { dyne }}{\mathrm{cm}}\right)\left(\frac{M_{\odot}}{M}\right)
$$

From the point of view of the membrane formalism the gravity of a black hole in equilibrium is produced by $p^{H}$.

The horizon's shear viscosity $\eta^{H}$ and the horizon's bulk viscosity $\zeta^{H}$ are correspondingly:

$$
\begin{gathered}
\eta^{H}=\frac{1}{16 \pi} \approx 10^{37} \frac{\mathrm{g}}{\mathrm{sec}}, \\
\zeta^{H}=-\frac{1}{16 \pi}=-10^{37} \frac{\mathrm{g}}{\mathrm{sec}} .
\end{gathered}
$$

Because the membrane paradigm regards a black hole as a two dimensional membrane with familiar mechanical properties it is rather easier to understand intuitively and compute quantitatively what happens with a black hole under some definite conditions. Let us consider a few examples.

If a black hole occurs as a result of gravitational collapse of an asymmetric celestial body (without rotation), then a nonspherical hole arises at the first moment. The hole's membrane is deformed and there is no balance between the surface pressure of the membrane and its gravity. So the membrane vibrates and radiates gravitational waves. The waves carry away the energy of the membrane deformation. This together with the membrane viscosity makes the horizon settle down into an absolutely spherical equilibrium shape.

Another example is a shape of the membrane of a rotating black hole. Centrifugal forces make a hole's membrane bulge out at its equator. The balance between the surface pressure, gravity and centrifugal forces determines the shape of the horizon's membrane.

Let us consider one very unusual property of the horizon's membrane. We emphasized above that the differential equations which describe the interaction of the horizon with the external universe 
are familiar physical laws (e.g, the Navier-Stokes equation and so on). But the solutions of the equations are determined also by the boundary conditions. In the case of standard physics the boundary conditions must be imposed at some initial moment or in the infinite past. That is not so for the hole's horizon! The point is that the horizon is a boundary between light-speed signals that can and those that cannot ever escape to spatial infinity. But this fact depends on the processes in the future, not in the past.

Whether a signal can escape depends on the region of spacetime to the future of the signal's source. It means that the motion of the horizon at any moment of time depends not on what has happened to the horizon in the past but what will happen to the horizon in the future.

This property can be illustrated by the problem of a free fall of a thin spherical shell of a matter of mass $\Delta M$ into a Schwarzschild hole with mass $M$. The spacetime geometry is that of Schwarzschild both interior to the shell and outside it. In the interior the Schwarzschild mass is $M$ and outside it is $M+\Delta M$. Now the light-speed signals with world lines at $r=2 M$ cannot be the boundary of the non-escape region because these signals and outgoing signals just outside $r=2 M$ will get caught and pulled into the hole by the added gravity of the shell when in the future the shell passes through them. The real boundary is generated by light-speed signals world lines of which are just outside of the surface $r=2 \mathrm{M}$. In the past, long before the shell arrives at the horizon this surface practically coincides with $r=2 M$. Then, as the shell nears it the surface (which is the real boundary, meaning the real horizon) starts to expand. This is because the world lines of its generators go farther an farther from $r=2 M$. This is their property in the Schwarzschild spacetime, and it does not depend on the approaching shell. When the shell finally passes through it, the added shell's gravity starts influence the motions of the generators of the surface, the horizon suddenly stops expanding and freezes at $r=2(M+\Delta M)$. These behaviors of the horizon are dictated by the properties of propagation of the light-speed signals which generate the horizon and which have the property to propagate at $r=2(M+\Delta M)$ after crossing with the shell. Thus, this behavior of the horizon before crossing with the shell (its expansion) depends on the events in the future (the crossing with the shell).

One refers to this dependence of future events as the "teleological" nature of the horizon (see Thorne et al. 1986). I would like to emphasize that these behaviors looks as if the hole's membrane lives in time which flows in the opposite direction: from the future into the past. Indeed in this case the change of the size of the horizon looks very natural and causal. If we accept this point of view, we should consider the extraction of the shell from the hole, and just after this extraction of the shell from the membrane at $r=2(M+\Delta M)$, the horizon starts contracting and settles down to $r=2 M$. We will see in Section 3 that this unusual property, namely, "feeling" information from the infinite future of the external observer, is a characteristic property not only of the horizon but also of the interior of a black hole.

\subsection{BLACK-HOLE ELECTRODYNAMICS}

A black hole horizon behaves as an electrically conducting sphere. To understand this let us ask what could be the external manifestation of the electric conductivity of a body in a flat spacetime. The simplest manifestation is the following. If one brings a positive electric charge close to a metal sphere then free electrons on the sphere's metal surface will be displaced with respect to the ions by the Coulomb electric forces. It polarizes the sphere. As a result, the electric field lines form a characteristic configuration in the space around the sphere. Now if one moves the charge parallel to the surface of the sphere from one position to another one, the characteristic configuration of the electric lines comes to a new place with some delay. This delay is determined by the resistivity of the sphere's metal surface. It turns out that if one brings a charge close to a non-spinning black hole, there is a similarity between the picture of the field lines in the vicinity of the black hole and the analogous picture in the vicinity of a metal sphere in a flat spacetime. Now the curvature of spacetime distorts the field line rather than displacement of real charges on the horizon. Nevertheless, it looks like the field of the charge polarizes the horizon.

If one moves the charge parallel to the hole's horizon to another position, then the configuration of the electric lines will settle down at the new place with some delay. Now it is determined by the finite time of propagation of electromagnetic signals. Nevertheless one can interpret it as an effective resistivity of the horizon. 
In general one can say that a horizon's membrane behaves as a metal sphere with a surface resistivity equal to $R_{H}=4 \pi \approx 377 \mathrm{ohms}$.

The membrane paradigm gives insight into possible behaviors of rotating black holes in interaction with magnetized plasma. We will draw an analogy with a dynamo. In its rotor the motion of wire coils in a magnetic field produces an electromotive force compelling the charges to flow through the conductor. A black hole is also a special dynamo of great size. If a spinning black hole is immersed in an external magnetic field, a powerful electric field will also develop in its vicinity. The magnetic field is created by the interstellar gas flowing into a black hole. The magnetic field lines will tend to rotate along with the spinning black hole. The motion of any magnetic field generates an electric field. In the case of a rapidly rotating, magnetized black hole, the electric field generated near its edges can produce an enormous voltage difference between the poles of the hole and its equatorial region:

$$
\Delta V \approx\left(10^{20} \text { volts }\right)\left(\frac{a}{M}\right)\left(\frac{M}{10^{9} M_{\odot}}\right)\left(\frac{B}{10^{4} G}\right),
$$

where $B$ is the magnetic field in the vicinity of the black hole. It is as though the spinning black hole was a huge battery. The electric field is responsible for accelerating the charged particles of the plasma and causing them to move along the magnetic lines of force. The total power output is

$$
P \approx\left(10^{45} \frac{\mathrm{erg}}{\sec }\right)\left(\frac{a}{M}\right)\left(\frac{M}{10^{9} M_{\odot}}\right)\left(\frac{B}{10^{4} G}\right),
$$

Probably this mechanism is the main "engine" of the active galactic nuclei.

\subsection{THERMODYNAMICS OF BLACK HOLES}

From many aspects of the thermodynamics of black holes, I will discuss the problem of the black hole's thermal quantum radiation and the related problem of the thermal atmosphere of a black hole.

S.Hawking (1974) claimed that a black hole should emit thermal radiation with temperature

$$
T_{H}=\frac{\hbar}{8 \pi k} M^{-1} \approx\left(10^{-7} K\right)\left(\frac{M_{\odot}}{M}\right) .
$$

How, in simple physical terms, could one understand that a black hole behaves like an ordinary body with temperature $T_{H}$. A key insight into thermal emission from a hole come from theoretical discoveries in the mid-1970s (see Unruh, 1976). The crucial point is the existence of the event horizon for some definite classes of observers. For example, an accelerated observer in an empty spacetime has a horizon. This observer cannot receive information from the region beyond the horizon. The virtual particles' vacuum fluctuation waves are not confined solely to the region above the horizon; part of each fluctuation wave is beyond the horizon and part is within the region which the observer can see. According to quantum mechanics this principle lack of information about vacuum fluctuation waves leads to the conclusion (for an accelerated observer) that they are real waves. As a result, this observer is bathed in a perfect bath of thermal radiation with temperature $T=\hbar a /(2 \pi k)$, where $a$ is the observer's acceleration. Since a static observer just above a Schwarzschild horizon can be viewed as analogous to an accelerated observer in flat spacetime with acceleration $a=c^{2} / z$, where $z$ is the distance from the horizon, such an observer should feel himself bathed in thermal radiation with local temperature $T=\hbar /(2 \pi k z)$. This thermal radiation forms a thermal atmosphere of the hole. The radiation, climbing up through the hole's gravitational field, would be redshifted by a factor $\left(1-\frac{2 M}{r}\right)^{1 / 2}$. It will emerge with temperature $T_{H}$. Most of the photons and other particles fly upward a short distance and are then pulled back down by the hole's enormous gravity. A few of the particles manage to escape the hole's gravitational grip and evaporate into space. These particles form the Hawking radiation.

Note that a free falling observer does not feel this thermal atmosphere. He "sees" only vacuum fluctuations to consist of pairs of virtual particles.

The process of the Hawking quantum evaporation is very slow. The total lifetime is proportional to the cube of $M$. For a 20 solar mass black hole it is $10^{70}$ years. 
In principle, the interactions of a black hole with the external Universe can change the process of extraction of the thermal energy from a black hole atmosphere drastically (see the review in referenced books).

\section{Physics inside a black hole}

\subsection{BLACK HOLE'S INTERIOR}

What can one say about the interior of a black hole?

This problem was the subject of a very active investigation last decades. There is a great progress in these researches. We know some important properties of the realistic black hole's interior, but some details and crucial problems are still the subject of much debate.

A very important point for understanding the problem of the black hole's interior is the fact that the path into the gravitational abyss of the interior of a black hole is a progression in time. We recall that inside a spherical hole, for example, the radial coordinate is timelike. It means that the problem of the black hole interior is an evolutionary problem. In this sense it is completely different: from a problem of an internal structure of other celestial bodies, stars for example.

In principle, if we know the conditions on the border of a black hole (on the event horizon), we can integrate the Einstein equations in time and learn the structure of the progressively more and more deep layers inside the black hole. Conceptually it looks simple, but there aro two types of principal difficulties which prevent realizing this idea consistently.

The first difficulty is the following. The internal structure of a general black hole, which rotates, even soon after its creation depends crucially on the conditions on the event horizon at tremendous future of the external observer (formally at the infinite future). It is because the light-like signal can propagate inside a black hole from the tremendous future to those regions if the regions are deep enough in the hole. The limiting light like signals which propagate from (formally) infinite future of the external observer form a border inside a black hole which is called a Cauchy horizon.

Thus, the structure of the regions inside a black hole depends crucially on the fate of the black hole at infinite future of an external observer. For example, it depends on the final state of the black hole evaporation, on possible collisions of the black hole with another black loole, and it depends on the fate of the Universe itself. It is clear that theoretitions feel themselves uncomfortable under such circumistances.

The second serious problem is related with existing of a singularity inside a black hole. Close to the singularity, where the curvatures of the spacetime approach Planck levels, the Classical General Relativity is not applicable. We have not the final version of the quantum theory of gravity yet, thus any extension of the discussion of physics in this region would be highly speculative. Fortunately, as we shall see, these singular regions are deep enough in the black hole interior and they are in the future with respect to overlying and preceding layers of the black hole where curvatures are not so high and which can be described by well-established theory.

The first attempts to investigate the interior of a Schwarzschild black hole have been made in the late 70's. It has been demonstrated that the parts of the interior of a black hole long after its formation and subsequent perturbations are virtually free of perturbations because the gravitational radiation from aspherical initial perturbations becomes infinitely diluted as it reaches these regions. But this result breaks down in general case when the angular momentum or the electric charge is not equal to zero. The reason for that is related with the fact that the topology of the interior of the rotating or/and charged black holes solution differs drastically from the Schwarzschild one. The key point is that the interior of this black hole possess a Cauchy horizon. This is a surface of infinite blueshift. Infalling gravitational radiation propagates inside the black hole along paths approaching the generators of the Cauchy horizon, and the energy density of this radiation will suffer an infinite blueshift as it approaches the Cauchy horizon.

In general the evolution with time into the black hole deeps looks like the following. There is a weak flux of gravitational radiation into a black hole through the horizon because of small perturbations outside of it. When this radiation approaches the Cauchy horizon it suffers an infinite blueshift. This infinite blueshift radiation together with the radiation scattered on the curvature of spacetime inside the black hole leads to formation of the curvature singularity of the spacetime along the Cauchy horizon. The infinite tidal gravitational forces arrise here. 
A detail discussion one can find in the following works: Poisson and Israel (1990), Ori (1992), Bonanno et al. (1995), Israel (1997) and references therein.

\subsection{QUANTUM EFFECTS}

In previous discussions we emphasized that the internal structure of black holes is a problem of the evolution in time starting from boundary conditions on the event horizon for all moment of time up to the infinite future of the external observer.

It is very essential to know boundary conditions up to infinity because we observed that the essential events: mass inflation and singularity formation, happened along the Cauchy horizon which brought information from the infinite future of the external spacetime. However, even an isolated black hole in the asymptotically flat spacetime cannot exist forever. It will be evaporated by Hawking quantum radiation. So far we discussed the problem without taking into account this ultimate fate of black holes. Even without going into details it is clear that quantum evaporation of the black holes is crucial for the whole problem.

What can we say about general picture of the black hole interior accounted quantum evaporation? To account quantum evaporation we have to change the boundary conditions on the event horizon as compared to the boundary conditions discussed above. Now they should include the flux of negative energy across the horizon which is related with the quantum evaporation. The last stage of the quantum evaporation when the mass of the black hole becomes comparable to the Planck mass, $m_{p l}=(\hbar / 6)^{1 / 2} \approx 2.2 \times 10^{-5} \mathrm{~g}$, is unknown. At this stage the spacetime curvature near the horizon reaches $l_{p l}^{-2}$, where $l_{p l}$ is the Planckian length, $l_{p l}=\left(G \hbar / c^{3}\right)^{1 / 2} \approx 1.6 \times 10^{-33} \mathrm{~cm}$. It means that from the point of view of the semi-classical physics a singularity arises here. Probably at this stage the black hole has characteristics of an extreme black hole, when an external event horizon and internal Cauchy horizon coincide.

As for the processes inside a true singularity in the black hole interior, they can be treated only in the framework of an unified quantum theory incorporating gravitation which is unknown.

\section{Astrophysics of black holes}

Do black holes exist in the Universe or are they only an abstract concept of the human mind? In principle, a black hole could be built artificially. However, this meets such grandiose technical difficulties that it looks impossible, at least in the immediate future. In fact, the artificial building of a black hole looks even more problematic than an artificial creation of a star. Thus we have to conclude that the physics of black holes, as well as the physics of stars, is the physics of celestial bodies. Stars definitely exist, but what may one say about the existence of astrophysical black holes?

Modern astrophysics deals with two types of black holes in the Universe:

1) stellar black holes, that is black holes of stellar masses, that were born when massive stars died; 2) supermassive black holes with masses up to $10^{9} M_{\odot}$ and greater at centers of galaxies $\left(M_{\odot}=\right.$ $2 \times 10^{33} \mathrm{~g}$ is the mass of the Sun).

These two types of black holes have been discovered. The third possible type of astrophysical black holes - primordial black holes will be discussed in subsection 4.5. Our main attention in Section 4 is focused on the possible observational manifestation of black holes.

\subsection{THE ORIGIN OF STELLAR BLACK HOLES}

"When all the thermo-nuclear sources of energy are exhausted a sufficiently heavy star will collapse" - this is the first phrase of the abstract of a remarkable paper by Oppenheimer and Snyder (1939). Every statement of this paper accords with ideas that remain valid today. The authors conclude the abstract by the following sentence: "... an external observer sees the star shrinking to its gravitational radius." This is the modern prediction of the formation of black holes when massive stars die.

How heavy should a star be to turn into a black hole? The answer is not simple. A star that is not massive enough ends up either as a white dwarf or a neutron star. There are upper limits on the masses of both these types of celestial bodies. For white dwarfs it is the Chandrasekhar limit, which is about $(1.2-1.4) \times M_{\odot}$. For neutron stars it is the Oppenheimer-Volkoff limit. The exact value 
of this limit depends on the equation of state at matter density higher than the density of nuclear matter $\rho_{0}=2.8 \times 10^{14} \mathrm{~g} \mathrm{~cm}^{-3}$. The modern theory gives for the maximal mass of a non-rotating neutron star the estimate $(2-3) \times M_{\odot}$. Rotation can increase maximal mass of a non-rotating neutron star only slightly up to $25 \%$. Thus one can believe that the upper mass limit for neutron stars should not be greater than $M_{0} \approx 3 M_{\odot}$. If a star at the very end of its evolution has mass greater than $M_{0}$ it must turn into a black hole. However this does not mean that all normal stars (on the "main sequence" of the Herzsprung-Russell diagram) with masses $M>M_{0}$ are black hole progenitors. The point is that the final stages of evolution of massive stars are poorly understood. Steady mass loss, catastrophic mass ejection and even disruption at supernovae explosions are possible. These processes can considerably reduce mass of a star at the end of its evolution. Thus the initial mass of black hole progenitors could be essentially greater than $M_{0}$.

There are different estimates for the minimal mass $M_{*}$ of a progenitor star that still forms a black hole. Uncertainty is $M_{*} \approx(10-40) M_{\odot}$ and even more. Note that the evolution of stars in close binary systems differs from the evolution of sole stars because of mass transfer from one star to another. The conclusions about masses of black hole progenitors in this case could be essentially different.

One can try to estimate how many black holes have been created by stellar collapse in our Galaxy during its existence. The estimates give the number of the order $10^{9}$.

\subsection{DISK ACCRETION ONTO BLACK HOLES}

For the purpose of finding and investigating black holes, two specific cases of accretion are of a particular importance: accretion in binary systems and accretion onto supermassive black holes that probably reside at the centers of galaxies. In both cases the accreting gas has big specific angular momentum. As a result the gas elements circle around the black hole in Keplerian orbits, forming a disk or a torus around it. Viscosity plays a crucial role for the accretion. It removes angular momentum from each gas element, permitting it to gradually spiral inward toward the black hole. At the same time the viscosity heats the gas, causing it to radiate. Probable sources of viscosity are turbulence in the gas disk and random magnetic fields. Unfortunately, we are not near to a good physical understanding of the effective viscosity. Large-scale magnetic fields can also play an important role in the physics of accretion.

The properties of the accreting disk are determined by the rate of gas accretion. An important measure of any accretion luminosity of a black hole is provided by the Eddington critical luminosity

$$
L_{E}=4 \pi G M_{h} \mu m_{p} c / \sigma_{T}=\left(1.3 \times 10^{38} \frac{\mathrm{erg}}{\mathrm{s}}\right) \mu\left(\frac{M_{h}}{M_{\odot}}\right) .
$$

Here $M_{h}$ is the mass of a black hole, $\mu$ is the molecular weight of electron, $m_{p}$ is the rest mass of the proton, and $\sigma_{T}$ is the Thomson cross section. It is the luminosity at which the radiation pressure just balances the gravitational force of the mass $M_{h}$ for a fully ionized plasma.

A useful measure of the accretion rate $\dot{M}$ is the so-called "critical accretion rate":

$$
\dot{M}_{E}=L_{E} c^{-2}
$$

where $L_{E}$ is given by equation (4.1). We shall use the dimensionless expression $\dot{m} \equiv \dot{M} / \dot{M}_{E}$.

The first models of the disk accretion were rather simple. They focused on the case of moderate rate of accretion $\dot{m}<1$. Subsequently theories for $\dot{m} \sim 1$ and $\dot{m} \gg 1$ were developed. They take into account complex processes in radiative plasma and various types of instabilities.

The source of luminosity for disk accretion is the gravitational energy that is released when gas elements in the disk spiral down. Most of the gravitational energy is released, generating most of the luminosity, from the inner parts of the disk. According to the theory for these simplest models the total luminosity of the disk is the following

$$
L \approx \begin{cases}\left(3 \times 10^{36} \frac{\mathrm{erg}}{\mathrm{s}}\right) \\ \left.3 \times 10^{37} \frac{\mathrm{erg}}{\mathrm{s}}\right)\left(\frac{\dot{M}}{10^{-9} M_{\odot} / \mathrm{yr}}\right), & \text { for a nonrotating hole }, \\ \left.\frac{\dot{M}}{10^{-9} M_{\odot} / \mathrm{yr}}\right), & \text { for a maximally rotating hole } .\end{cases}
$$

The accretion rate $\dot{M}$ is an arbitrary external parameter, which is determined by the source of gas (for example, by the flux of gas from the upper atmosphere of the companion star in a 
binary system). We normalized $\dot{M}$ by the value $\dot{M}_{0}=10^{-9} M_{\odot} / \mathrm{yr}$ because this is probably the typical rate at which a normal star is dumping gas onto a companion black hole. In this model the accretion gas is assumed to be relatively cool, with its temperature much less than the virial temperature corresponding to the potential energy in the gravitational field. As estimates show, a geometrically thin disk (with heights $h \ll r$ ) might be formed under these conditions. This is the so-called standard disk model. In this model the electron and ion temperatures are equal, and the disk is effectively optically thick. The temperature of the gas in the inner parts of the disk reaches $T \approx 10^{7}-10^{8} \mathrm{~K}$. In this region electron scattering opacity modifies the emitted spectrum so that it is no longer the blackbody spectrum. Instead, the total spectrum of the disk radiation is a power law $F \sim \omega^{1 / 3}$ with an exponential cut off at high frequencies. The innermost regions of such "standard" disks are probably unstable. The thin accretion disk model is unable to explain the hard spectra observed in accretion flows around black holes in many observable cases.

A few types of hot accretion flow models have been proposed. Among them a model with a hot corona above a standard thin accretion disk. In another model the ions in the inner region are hot $T_{i} \approx 10^{11} \mathrm{~K}$ but the electrons are considerably cooler $T_{e} \approx 10^{9} \mathrm{~K}$. This inner disk is thicker than in the "standard" model and produces most of the $X$-ray emission. The models with hot ions and cooler electrons are optically thin.

Further development of the theory of disk accretion led to more sophisticated models. It have been demonstrated that when the luminosity reaches the critical one (corresponding to $\dot{m} \equiv \dot{M} / \dot{M}_{E}$ of the order of unity) radiation pressure in the inner parts of the disk dominates the gas pressure and the disk is thermally and viscously unstable. For especially big $\dot{m}>80$ the essential part of the energy of the plasmais lost by advection into the black hole horizon because the radiation is trapped in the accretion gas and is unable to escape. This process stabilizes the gas flow against perturbations. Advection can also be important for smaller $\dot{m}$. For high mass accretion rates the height of the accretion disk becomes comparable to its radius. In modern models the radial pressure gradients and the motion of gas elements along radius are taken into account. In the innermost parts of the disk and down to the black hole the flow of gas is supersonic.

Recently, a new class of optically thin hot disk solutions has been discovered. In this model the most of the viscously dissipated energy is advected with the accreting gas, with only a small fraction of the energy being radiated. It is because the gas density is so low that the radiative efficiency is very poor. These models were named advection-dominated. They have been applied successfully to a few concrete celestial objects.

In conclusion we note that in some models of disk accretion electron-positron pair production can be important. We believe that new models involving recent achievements of plasma physics will play a key role in the modern astrophysics of black holes.

\subsection{EVIDENCE FOR BLACK HOLES IN STELLAR BINARY SYSTEMS}

Probably the best evidence that black holes exist comes from studies of $X$-ray binaries. The arguments that are used to prove that an $X$-ray binary contains a black hole, are as follows:

1. The $X$-ray emitting object in a binary system is very compact, and therefore cannot be an ordinary star. Thus it is either a neutron star or a black hole. This argument comes mainly from analysis of the features of emitted $X$-rays.

2. Analysis of the observational data allows one to determine the orbital motion in the binary system makes it possible to obtain the mass of the compact object. The data on the observed velocity of the optical companion star is of the most importance. Note that the Newtonian theory is always sufficient for the analysis. The technique of weighing stars in binaries is well known in astronomy. If the mass of the compact component is greater than the maximal possible mass of neutron stars $M_{0} \approx 3 M_{\odot}$ (see Section 4.1), then it is a black hole.

It is worth noting that this evidence is somewhat indirect because it does not confront us with the specific relativistic effects that occur near black holes and which are peculiar to black holes alone. However, it is the best that modern astronomy has proposed so far. In spite of these circumstances, we believe that the logic of the arguments is reliable enough.

According to the generally accepted interpretation, we have the necessary observational confirmation only for a few systems at the present time. For these systems, we have strong reasons to 
TABLE 1. Black-hole candidates in binary systems [Cherepashchuk (1996)].

\begin{tabular}{|c|c|c|c|c|c|}
\hline System & $\begin{array}{l}\text { Spectral type } \\
\text { of the optical } \\
\text { companion }\end{array}$ & $\begin{array}{l}\text { Orbital } \\
\text { period } \\
\text { (days) }\end{array}$ & $\begin{array}{l}\text { Mass of the } \\
\text { compact } \\
\text { companion } \\
\text { (in } M_{\odot} \text { ) }\end{array}$ & $\begin{array}{l}\text { Mass of the } \\
\text { optical } \\
\text { companion } \\
\text { (in } M_{\odot} \text { ) }\end{array}$ & $\begin{array}{r}X \text {-ray } \\
\text { lunuinosity } \\
(\mathrm{erg} / \mathrm{sec})\end{array}$ \\
\hline $\begin{array}{l}\text { Cyg X-1 } \\
\text { (V } 1357 \text { Cyg) }\end{array}$ & O9.7Iab & 5.6 & $7-18$ & $20-30$ & $\sim 8 \times 10^{37}$ \\
\hline LMC X-3 & B(3-6)II-III & 1.7 & $7-11$ & $3-6$ & $\sim 4 \times 10^{38}$ \\
\hline LMC X-1 & $O(7-9) \mathrm{III}$ & 4.2 & $4-10$ & $18-25$ & $\sim 2 \times 10^{3+8}$ \\
\hline $\begin{array}{l}\text { A0620-00 } \\
\text { (V616 Mon) }\end{array}$ & $K(5-7) \mathrm{V}$ & 0.3 & $5-17$ & $\sim 0.7$ & $\leq 10^{3 R}$ \\
\hline $\begin{array}{l}\text { GS } 2023+338 \\
\text { (V } 404 \mathrm{Cyg} \text { ) }\end{array}$ & KorV & 6.5 & $10-15$ & $0.5-1.0$ & $\leq 6 \times 10^{38}$ \\
\hline $\begin{array}{l}\text { GRS 1121-68 } \\
\text { (XN Mus 1991) }\end{array}$ & $K(3-5) V$ & 0.4 & $9-16$ & $0.7-0.8$ & $\leq 10^{38}$ \\
\hline $\begin{array}{l}\text { GS } 2000+25 \\
(\mathrm{QZ} \mathrm{Vul})\end{array}$ & $\mathrm{K}(3-7) \mathrm{V}$ & 0.3 & $5.3-8.2$ & $\sim 0.7$ & $\leq 10^{38}$ \\
\hline $\begin{array}{l}\text { GRO J0422+32 } \\
\text { (XN Per 1992= } \\
=\text { V518 Per) }\end{array}$ & $\mathrm{M}(0-4) \mathrm{V}$ & 0.2 & $2.5-5.0$ & $\sim 0.4$ & $\leq 10^{38}$ \\
\hline $\begin{array}{l}\text { GRO J1655-40 } \\
\text { (XN Sco 1994) }\end{array}$ & F5IV & 2.6 & $4-6$ & $\sim 2.3$ & $\leq 10^{38}$ \\
\hline XN Oph 1977 & K3 & 0.7 & $5-7$ & $\sim 0.8$ & $\leq 10^{38}$ \\
\hline
\end{tabular}

believe that the compact $X$-ray emitting companions are black holes. Some characteristics of these leading black hole candidates are summarized in Table 1 [according to Cherepashchuk (1996)].

The most plausible masses of compact objects in these systems are considerably larger than $M_{0} \approx 3 M_{\odot}$. The strongest candidate are those which have a dynamical lower limit of the mass of the compact object (or so-called mass function $f(M)$ ) greater than $3 M_{\odot}$. ¿From this point of view the strongest candidates are GS $2033+338\left(\mathrm{f}(\mathrm{M})=6.5 M_{\odot}\right)$, GS $2000+25\left(\mathrm{f}(\mathrm{M})=5 M_{\odot}\right)$, and XN Oph $1977\left(\mathrm{f}(\mathrm{M})=4 M_{\odot}\right)$.

The total number of systems that are frequently mentioned as possible candidates for black holes of stellar mass is about 20. All seriously discussed candidates are $X$-ray sources in binary systems. Some of them are persistent, other are transient. Begelman and Rees (1996) summarize the present status as follows: "There is also overwhelming evidence for black holes in our own galaxy, formed when ordinary massive stars die, each weighting a few times as much as the Sun". Most of experts now agree with this unambiguous conclusion.

During the more than 25 years since the discovery of the first black hole candidate Cyg X-1 only a few new candidates have been added. This is in contrast to the rapid increase of the number of identified neutron stars. At present many hundreds of neutron stars have been identified in the Galaxy. About 100 of them are in binary systems. One might conclude that black holes in binary systems are exceedingly rare objects. This is not necessarily true, however. The small number of identified black hole candidates may as well be related to the specific conditions which are necessary for their observable manifestation. 
According to estimations the evolutionary stage when a black hole binary continuously radiates $X$-ray may last only $10^{4}$ years. We can thus detect it only during this short period. In effect, the population of black-hole binaries may be much larger than what we can presently observe. Such systems may be as common as neutron star binaries.

\subsection{SUPERMASSIVE BLACK HOLES IN GALACTIC CENTERS}

Since the middle of this century astronomers have come across many violent or even catastrophic processes associated with galaxies. These processes are accompanied by powerful releases of energy and are fast not only by astronomic but also by earthly standards. They may last only a few days or even minutes. Most such processes occur in the central parts of galaxies, the galactic nuclei.

About one percent of all galactic nuclei eject radio-emitting plasma and gas clouds, and are themselves powerful sources of radiation in the radio, infrared, and especially, the "hard" (short wavelength) ultraviolet, $\mathrm{X}$-ray and gamma regions of the spectrum. The full luminosity of the nucleus is in some cases $L \approx 10^{47} \mathrm{erg} / \mathrm{s}$, and millions of times the luminosity of the nuclei of more quiet galaxies, such as ours. These objects were called active galactic nuclei (AGN). Practically all the energy of activity and of the giant jets released by galaxies originates from the centers of their nuclei.

Quasars form a special subclass of AGN. Their characteristic property is that their total energy release is hundreds of times greater than the combined radiation of all the stars in a large galaxy. At the same time the average linear dimensions of the radiating regions are small: a mere one-hundredmillionth of the linear size of a galaxy. Quasars are the most powerful energy sources registered in the Universe to date. What processes are responsible for the extraordinary outbursts of energy from AGN and quasars?

Learning about the nature of these objects involves measuring their sizes and masses. This is not easy at all. The central emitting regions of AGN and quasars are so small that telescope view reveals them just as point sources of light. Fortunately quite soon after the discovery of the quasar 3C 273 it was shown that its brightness changed. Sometimes it changes very rapidly, in less than a week. After this discovery, even faster variability (at timescale of a few hours or less) were detected in other galactic nuclei. From these variations one could estimate the dimensions of the central parts of the nuclei that are responsible for radiation. The conclusion was that these regions were not more than a few light-hours in diameter. That is, they are comparable to the solar system in size.

In spite of the rather small linear dimensions of quasars and many galactic nuclei, their masses turned out to be enormous. They were first estimated using formula (4.1). For quasistatic objects the luminosity cannot be essentially greater than $L_{E}$. A comparison of the observed luminosity with the expression (4.1) gives an estimate of the lower limit of the central mass. In some quasars this limit is $M \approx\left(1-10^{2}\right) \times 10^{7} M_{\odot}$. These estimates are supported by data on the velocities within the galactic nuclei of stars, and gas clouds accelerated in the gravitational fields of the center of the nuclei. We will discuss this in the end of this Section.

Great mass but small linear dimensions prompt the guess that there could be a black hole. This would account for all the extraordinary qualities of these objects. Now it is generally accepted that in AGN there are supermassive black holes with accretion gas (and maybe also dust) disks. One of the most important facts implied by observations, especially by means of radio telescopes, is the existence of directed jets from the nuclei of some active galaxies. For some of the objects there are evidence that radio components move away from the nucleus at ultrarelativistic velocities. The existence of an axis of ejection strongly suggests the presence of some stable compact gyroscope, probably a rotating black hole. In some cases one can observe evidence that there is also precession of this gyroscope. An essential role in the physics of processes in the centers of AGN is probably played by black-hole electrodynamics.

In the model of a supermassive black hole with an accretion disk for AGN one requires sources of fuel - gas or dust. The following sources have been discussed: gas from nearby galactic companion (the result of interaction between the host galaxy and the companion), interstellar gas of the host galaxy, disruption of stars by high velocity collisions in the vicinity of a black hole, disruption of stars by the tidal field of the black hole and some others. 
Clearly, the processes taking place in quasars and other galactic nuclei are still a mystery in many respects. But the suggestion that we are witnessing the work of a supermassive black hole with an accretion disk seems rather plausible. Rees (1990) advocates a hypothesis that the massive black holes are not only in the active galactic nuclei but, also in the centers of "normal" galaxies (including nearby galaxies and our own Milky Way). They are quiescent because they are now starved of fuel (gas). Observations show that galactic nuclei were more active in the past. Thus, "dead quasars" (massive black holes without fuel) should be common at the present epoch.

How can these black holes be detected? It has been pointed out that black holes produce cusplike gravitational potentials and hence they should produce cuspy-like density distributions of the stars in the central regions of galaxies. Some authors have argued that the brightness profiles of the central regions of particular galaxies imply that they contain black holes. However the arguments based only on surface brightness profiles are inconclusive. The point is that a high central number density of stars in a core with small radius can be the consequence of dissipation, and a cusp-like profile can be the result of anisotropy of the velocity dispersion of stars. Thus these properties taken alone are not sufficient evidence for the presence of a black hole.

The reliable way to detect black holes in the galactic nuclei is analogous to the case of black holes in binaries. Namely, one must prove that there is a large dark mass in a small volume, and that it can be nothing other than a black hole. In order to obtain such a proof we can use arguments based on both stellar kinematics and surface photometry of the galactic nuclei.

If the distribution of the mass $M$ and the luminosity $L$ as functions of the radius are known we can consider the mass-to-light ratio $M / L$ (in solar units) as a function of radius. This ratio is well known for different types of stellar populations. As a rule this ratio is between 1 and 10 for elliptica.l galaxies and globular clusters (old stellar population dominates there). If for some galaxy the ratio $M / L$ is almost constant at rather large radii (and has a "normal" value between 1 and 10) but rises rapidly (toward values much larger than 10) as one approaches the galactic centre, then there is evidence for a central dark object (probably a black hole).

As an example consider galaxy NGC 3115 which is at a distance of $9.2 \mathrm{Mpc}$ from us [Kormendy and Richstone (1992)]. For this galaxy $M / L \approx 4$ and almost constant over a large range of radii $r>4^{\prime \prime}$ (in angular units). This value is normal for a bulge of this type of galaxy. At radii $r<2^{\prime \prime}$ the ratio $M / L$ rises rapidly up to $M / L \approx 40$. If this is due to a central dark mass added to a stellar distribution with constant $M / L$, then $M_{H}=10^{9.2 \pm 0.5} M_{\odot}$.

Is it possible to give another explanation of the large mass-to-light ratio in the central region of a galaxy? We cannot exclude the possibility that a galaxy contains a central compact cluster of dim stars. But it is unlikely. The central density of stars in the galaxy NGC 3115 is not peculiar. It is the same as in the centers of globular clusters. The direct observational data (spectra and colors) of this galaxy do not give any evidence of a dramatic population gradient near the center. Thus, the most plausible conclusion is that there is a central massive black hole.

Unfortunately, it is difficult to detect massive black holes in giant elliptical galaxies with active nuclei, where we are almost sure black holes must exist because we observe their active manifestation [Kormendy (1993)]. The reason for this is a fundamental difference between giant elliptical galaxies (the nuclei of some of them are among the most extreme examples of AGN), dwarf elliptical galaxies and spiral galaxies. Dwarf ellipticals rotate rapidly and star velocity dispersions are nearly isotropic. Giant elliptical galaxies do not rotate significantly and they have the anisotropic velocities. It is not so easy to model these dispersions. Furthermore, giant elliptical galaxies have large cores and shallow brightness profiles. Consequently, the projected spectra are dominated by light from large radii, where a black hole has no effect.

The technique described above has been used to search for black holes in galactic nuclei. Another possibility is to observe rotational velocities of gas in the vicinity of the galactic center. So far (the middle of 1997) black hole detections have been reported for the following galaxies: M32, M31, NGC 3377, NGC 4594, Milky Way, NGC3115, M87, and NGC 4258 [for review see Kormendy (1993), see also Miyoshi et al. (1995) Kormendy and Richstone (1992)]. Some evidence for a supermassive black hole in NGC 4468B was reported by Kormendy et al. (1997).

Special investigations were performed in the case of the galaxy M87 [see Dressler (1989) for review of earlier works and Lauer et al. 1992]. This is a giant elliptical galaxy with active nucleus and a jet from the center. At present there is secure stellar-dynamical evidence for a black hole with mass $M \approx 3 \cdot 10^{9} M_{\odot}$ in this galaxy. The Hubble Space Telescope has revealed a rotating 
TABLE 2. Estimated masses of black holes in galactic nuclei.

\begin{tabular}{|l|l|}
\hline Galaxy & Mass of black hole (in $\left.M_{\odot}\right)$ \\
\hline M31 & $2 \times 10^{7}$ \\
M32 & $(2-5) \times 10^{6}$ \\
Milky Way & $3 \times 10^{6}$ \\
NGC 4594 & $10^{9}$ \\
NGC 3115 & $10^{9}$ \\
NGC 3377 & $10^{8}$ \\
M87 & $2.4 \times 10^{9}$ \\
NGC 4258(M106) & $3.6 \times 10^{7}$ \\
& \\
\hline
\end{tabular}

disk of gas orbiting the central object in the galaxy [Ford et al. (1994), Harms et al. (1994)]. The estimated mass of the central object is $M=2.4 \times 10^{9} M_{\odot}$. The presence of a black hole in M87 is especially important for our understanding of the nature of the central regions of galaxies because in this case we observe also the activity of the "central engine".

Radio-astronomical observations of the nucleus of the galaxy NGC 4258 are of special interest [Miyoshi et al. (1995)]. Using radio interferometry technique of observation of maser lines of molecules of water in gas clouds orbiting in the close vicinity of the nucleus, the observers obtained the angular resolution 100 times better that in the case of observations by the Hubble Space Telescope. The spectral resolution is 100 times better as well. According to the interpretation of the observations the center of NGC 4258 harbors a thin disk which was measured on scales of less than one light-year. The mass of the central object is $3.6 \times 10^{7} M_{\odot}$. According to the opinion of Begelman and Rees (1996): "It represents truly overwhelming evidence for a black hole... NGC 4258 is the system for which it is hardest to envisage that the mass comprises anything but a single black hole".

In conchusion we list in Table 2 estimates of masses of black holes in the nuclei of some galaxies [Kormendy (1993), van der Marel (1995), Miyoshi et al. (1995)].

Progress in this field is very rapid and in the nearest future our knowledge about evidence of supermassive black holes in galactic nuclei will be more profound.

\subsection{PRIMORDIAL BLACK HOLES}

Modern astrophysics considers also the third possible type of black holes in the Universe - primordial black holes. These black holes might appear from inhomogeneities at the very beginning of the expansion of the Universe. Their masses can be arbitrary, but primordial black holes with $M \leq 10^{15} \mathrm{~g}$ would have radiated away their mass by the Hawking quantum process in a time $t \leq 10^{10}$ years (the age of the Universe) Only primordial black holes with mass $M>10^{15} \mathrm{~g}$ could exist in the contemporary Universe.

Searches for PBHs attempt to detect a diffuse photon (or another particle) background from a distribution of $\mathrm{PBH}$ or to search directly for the final emission stage of individual black holes. Using the theoretical spectra of particles and radiation emitting by evaporating black holes of different masses, one can calculate the theoretical backgrounds of photons and other particles produced by a distribution of PBHs emitting over the lifetime of the Universe. The level of this background depends on the integrated density of $\mathrm{PBHs}$ with initial masses in the considered range.

A comparison of the theoretical estimates with the observational cosmic ray and $\gamma$-ray backgrounds place an upper limit on the integrated density of $\mathrm{PBHs}$ with initial masses in this range. According to estimates of MacGibbon and Carr (1991), this limit corresponds to $\approx 10^{-6}$ of the integrated mass density of the visible matter in the Universe (matter in the visible galaxies). The comparison of the theory with other observational data gives more weak limits (for review see Halzen et al. 1991, Coyne 1993).

The search for high energy gamma-ray bursts as direct manifestation of the final emission of 
the evaporating (exploding) individual PBHs has continued for more than 20 years. No positive evidence for existence of PBHs has been reported (see Cline and Hong $(1992,1994)$.

A population of $\mathrm{PBHs}$ whose influence is small today may have been more important in the earlier epochs of the evolution of the Universe. Radiation from PBHs could perturb the usual picture of cosmological nucleosynthesis, distort the microwave background and produced too much entropy in relation to the matter density of the Universe. Limits on the density of PBHs, now or at earlier times, can be used to provide information on the homogeneity and isotropy of the very early Universe, when they were formed. For review see Novikov et al. (1979), Carr et al. (1994).

The final state of the black hole evaporation is still unclear. There is a possibility that the endpoint of the black hole evaporation is a stable relic. The possible role of such relics in cosmology was first discussed by MacGibbon (1987), for the recent review see Barrow et al. (1992).

\section{Conclusions}

Black holes are absolutely unusual objects. In spite of all the progress the nature of space and time in the black holes remains to a large extent a mystery for us. Some aspects of the problem still look as scientific toys interesting only for specialists.

As for the practical realization of new ideas, I would like to conclude the paper with the remaining that in the middle of the last century even such a practical (now!) thing as electricity looked as a scientific toy. When at that period Mr. Gladstone asked Faraday about the practical worth of electricity, M.Faraday answered: "One day, Sir, you may tax it".

I am an optimist and believe in enormous promise of the new field of research concerned physics and astrophysics of black holes.

\section{References}

Barrow, L.D., Copeland E.J. and Liddle A.R. (1982) Phys. Rev. D51, 1741.

Begelman, M.C. and Rees M.I. (1996) Gravity's Fatal Attraction: Black holes in the Universe (Scientific American Library) W.H. Freeman.

Bonanno, A., Droz. S., Israel, W. \& Morsink, S. (1995) Proc. Roy. Soc A450 553.

Carr, B.J., Gilbert J. H., and J. E. Lidsey J. E. (1994) Phys. Rev. D50, 4853.

Cherepashchuk, A.M. (1996) Russian Physics - Uspekhi, 39, 759.

Cline, D.B. and Hong W. (1992) Astrophys, J, 401, L57.

Cline, D.B. and Hong W.(1994) BAAS 185, 116.

Coyne, D.G. (1993) in International symposium on black holes, membranes, wormholes and superstrings. (Singapore, World Scientific Publishing Co.), ed. by S. Kalara and D. V. Nanapoulos, p. 159.

Dresler, A. (1989) in D. E. Osterbrock and J. S. Miller (eds), IAU Symposium 134, Active Galactic Nuclei, Kluwer, Dordrecht, p.217.

Ford, H. C., Harms R. J., Tsvetanov Z. I., Hartig G. F., Dressel L. L., Kriss G. A., Bohlin R. C., Davidsen A. F., Margon B., and Kochhar A. K. (1994) Astrophys, J, 435, L27.

Frolov, V.P. andNovikov I.D. (1998) Black Holes Physics: Basic concept and New Developments, Kluwer Acad. Publ., in preparation.

Halzen, F., Zas E., MacGibbon J. H., and Weekes T. C. (1991) Nature 353, 807.

Harms, R. J., Ford H. C., Tsvetanov Z. I., Hartig G. F., Dressel L. L., Kriss G. A., Bohlin R. C., Davidsen A. F., Margon B., and Kochhar A. K. (1994) Astrophys, J, 435, L35.

Hawking, S.W. (1974) Nature, 248, 30.

Israel, W. (1997) in the book Relativistic Astrophysics ed. Jones B. and Markovic D., Cambridge University Press, p.173.

Kawaler, S.D., Novikov I.,Srinivasan G. (1997) Stellar Remnants, Saas-Fee Advanced Course 25, Springer, p. 237.

Kormendy, J. (1993) In The Nearest Active Galaxies, Eds. J.E. Beckman, H. Netzer, and L. Colina (Consejo Superior de Investigaciones Cientificas, Madrid), p. 197.

Kormendy, J. and Richstone D. (1992) Astrophys. J. 393, 559.

Kormendy, J., Bender R., Maggorian J., Tremaine S., Gebhardt K., Richstone D., Dressler A., Faber S. M., Grillmair C., and Lauer T. R. (1997) astro-ph/9703188, $27 \mathrm{Mar} 97$.

Lauer, T. R. et al. (1992) Astronomical J. 103, 703.

MacGibbon, J.H. (1987) Nature 329, 308.

MacGibbon, J.H. andCarr B.J. (1991) Astrophys. J. 371, 447.

Miyoshi, M., Moran J., Herrnstein J., Greenhill L., Nakai N., Diamond P., and M. Inoue M. (1995) Nature, $373,127$.

Novikov, I.D. and Frolov V.P. (1989) Physics of Black Holes. Kluwer Acad. Publ. (Russian version (19 86, Nauka, Moscow). 
Novikov, I. D., A. G. Polnarev A. G., Starobinsky A. A. and Zeldovich Ya. B. (1979) Astron. and Astrophys. $80,104$.

Oppenheimer, J.R. and Snyder H. (1939) Phys. Rev. 56, 455.

Ori, A. (1992) Phys. Rev. Lett. 68, 2117.

Poisson, E., \& Israel, W. (1990) Phys. Rev. D41, 1796.

Rees, M. (1990) Science 247, N4944, 16 February, p.817.

ndent Thorne, K.S., Price R.H. and Macdonald D.A. (1986) Black Holes: The Membrane Paradigm, Yale Univ. Press, New Haven.

van der Marel, R.P. (1995), In I.Appenzeller (ed.), Highlights of Astronomy, 10, p.527. 